\title{
Vehicle Aerodynamic Stability Analysis under High Crosswinds
}

\author{
Aleksander Grm* - Milan Batista \\ University of Ljubljana, Faculty of Maritime Studies and Transport, Slovenia
}

In a strong crosswind, wind direction change may result in vehicle stability loss. This paper presents a numerical study of vehicle aerodynamic stability in a high crosswind situation. We start with a model explanation and introduce the complete computational fluid dynamics (CFD) framework used in the study. Important CFD parameters such as mesh type, turbulence model, and boundary conditions are exposed and discussed in detail. We demonstrate and discuss the flow structure around a simplified truck model. Results of the CFD analysis are compared to experimental data, showing an almost perfect match. The final CFD outcomes are functions of aerodynamic coefficients that depend on the apparent wind angle. Then, CFD results are used in the application of an aerodynamic stability analysis for the truck model. Finally, the critical stability bounds are calculated, showing the marginal crosswind driving properties of the vehicles.

Keywords: aerodynamic stability/ instability, computational wind engineering, overturning moments, computational fluid dynamics, wind-structure interaction

Highlights

- A CFD vehicle aerodynamic stability analysis is presented in detail.

- Aspects of the effect of mesh type on the aerodynamics coefficients in CFD calculations are presented.

- A few highlights of the fit procedure for aerodynamic coefficients are emphasized.

- Rotation, rollover, and side-slip stability regions are shown in retrospect.

\section{O INTRODUCTION}

The flow around a heavy vehicle exhibits complex characteristics, such as a turbulent boundary layer, separation and reattachment on the vehicle surface, massive separation at the rear part of the vehicle, shear layer evolution, and large wakes. Furthermore, the Reynolds number $(R e)$ is sufficiently high that turbulent flow develops almost everywhere, except during the early stages of its collision with the vehicle. Understanding the effects of these flow characteristics on vehicles aerodynamic properties has considerable practical importance. Numerous studies have been conducted both in academia and industry to understand and control the flow around the heavy vehicles [1].

The study of three-dimensional flow around a ground vehicle has become a subject of significant importance in many areas. For example, in the study of vehicle aerodynamic stability in a crosswind situation [2] and [3] when the aerodynamic coefficients depend on the apparent wind direction. One significant problem is the accuracy of the aerodynamic coefficients. The flow around ground vehicles, including the vehicle covered in this work, shares unsteady and three-dimensional features with that around other aerial or marine transportation vehicles. However, in contrast to the flow around a commercial airplane, ship, or submarine, a key phenomenon in the flow around a ground vehicle is massive flow separation, which significantly affects the aerodynamic forces and moments experienced by the vehicle. This phenomenon is more pronounced for heavy vehicles that have a boxy shape with many sharp edges, designed for carrying as much cargo and as many passengers as possible within regulated external dimensions.

Analysis of such complex flow can be performed through the experiments or numerical investigation. Currently, experiments with various vehicles are not always feasible due to the high costs of wind tunnel experiments, especially for a 1:1 model. The alternatives are computational fluid dynamics (CFD) tools. In the past, full 3D vehicle CFD analysis was significantly limited by the lack of computational resources and the high precision of CFD turbulent algorithms. Currently, however, this is no longer the case, and we can compute numerical calculations for almost any vehicle in reasonable time with affordable hardware. In this article, we used an ANSYS-CFX system to calculate the aerodynamic coefficients.

The article describes our model, including details on the geometry setup, computational domain, mesh generation, CFX setup, boundary conditions, and stop criteria. We have paid significant attention to the analysis of aerodynamic coefficients with respect to the mesh type. We focused on the difference in the boundary layer mesh algorithms. As described herein, the classical boundary layer meshing algorithm (first layer thickens (FLT)) approach did 
not work as expected. Instead, an alternative meshing algorithm (first aspect ratio (FAR)) showed very good convergence results. After the model section, this article describes the definition of aerodynamic coefficients. The main idea behind is to obtain useful fitting models [4] to [7] that optimally represent the aerodynamic coefficients with respect to apparent wind direction and yaw angle $\psi$. A complex fit model does not always have better fitting properties. The ability to mimic a physical background in the fit model results in good fit model properties for real life data. As described in the results subsection, a linear combination of basic functions, fit model Eq. (3), shows most of the physical interpretation of the nature of aerodynamic coefficients with respect to the yaw angle $\psi$.

In the last section, the application of the fit model to vehicle aerodynamic stability is presented. Estimates are calculated for critical rollover, rotation, and side-slip parameters.

\section{METHODS}

The analysis of turbulent flow around a vehicle requires great care when building the CFD model. In order to obtain the necessary aerodynamic coefficients, a numerical model capable of producing a solution independent of time-step size, convergence limits, and other pertinent modeling conditions must be developed. In this study, a full three-dimensional CFD simulation model is used, because the vehicle is rotated around the center of gravity (CG), as shown in Fig. 1. The three-dimensional CFD model is developed using the ANSYS 16.0 workbench. CFX is used to solve the steady Reynolds-averaged Navier-Stokes equations, using the finite volume method with a two-equation turbulence model of the shear stress transport (SST) model [9], as is typically recommended for use in the calculation of aerodynamic coefficients [3]. We will not describe the details of the physical model of turbulent fluid flow, as it is completely described in the literature [10] to [12].

The modeled geometry is a truck model, described in [8] and shown in Fig. 1. The CAD model is identical to the model used in the wind tunnel experiments referenced in [5] and [8]. In this way, simulation data can be validated with experimental data. The top parts of Fig. 1 and Fig. 3 show the placement of the coordinate system and angle of rotation. The axis of rotation is parallel to the $z$ axis and passes through CG (Fig. 3). In external aerodynamics, proper mesh construction that embeds specific flow conditions plays a significant role. In real life, the flow conditions are turbulent and the Reynolds number $(R e)$ [12] is very high $\left(R e \geq 10^{6}\right)$.

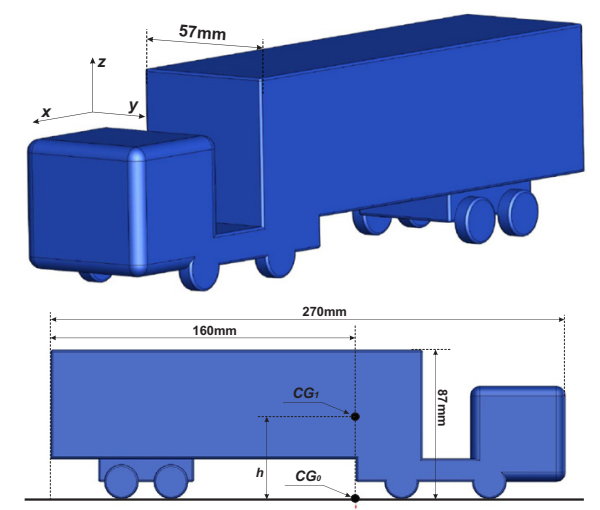

Fig. 1. Truck geometry constructed in SolidWorks [8]; cabin edge fillet is $5 \mathrm{~mm}$, rest edge fillet is $1 \mathrm{~mm}$

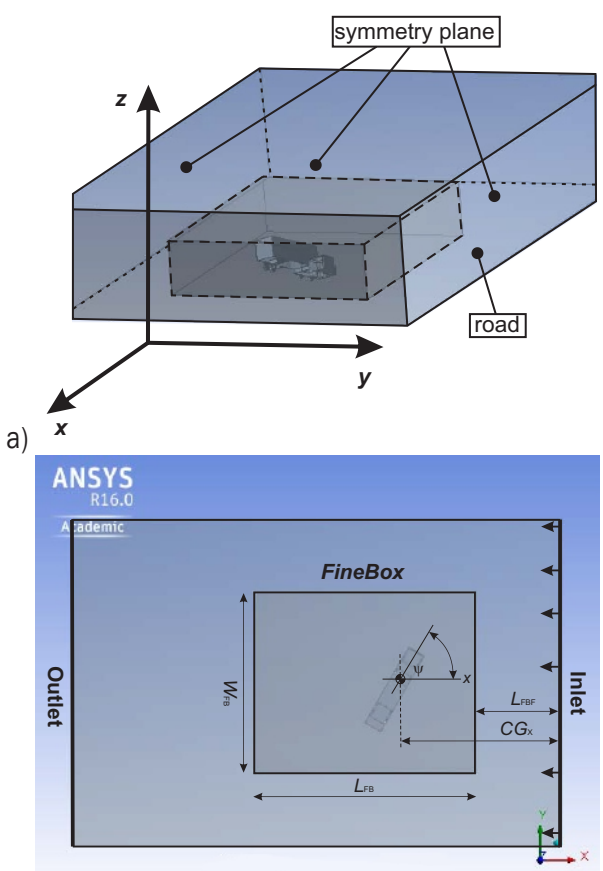

b)

Fig. 2. Domain configuration; a) 3D domain model with clear view of FineBox subdomain surrounding truck; $b$ ) z projection of the domain

Turbulent flow develops almost everywhere, except in the zone where the flow first collides with the vehicle. To properly capture turbulent flow, the mesh must be constructed such that the mesh size detects the turbulent flow properties predicted by the turbulent SST model [9]. The mesh size will also define the computational time, and therefore, the mesh must be reasonably sized. External aerodynamics 
must cope simultaneously with two flow regions. The first mesh-related characteristic flow region is the boundary layer region [13]. In the boundary layer region, the mesh must be constructed to account for turbulent model properties (wall functions [12]), according to the turbulent SST model. In the second region, which is referred to as the FineBox region and is positioned near the vehicle, the mesh must be fine enough to detect vortices that develop in the vicinity of the vehicle. The FineBox region forms a subdomain of the computational domain, and its placement can be seen in Fig. 2. The dimensions of the FineBox subdomain are related to the vehicle length $(L)$. In the simulation, we define the following set of dimensions for the FineBox subdomain: width $(W F B)$, length $(L F B)$, front length $(L F B F)$, position of the center of gravity in the $x$ direction $\left(C G_{x}\right)$, and height $(H F B)$. Most references for aerodynamics calculations suggest fixing the FineBox dimensions (labels are explained at Fig. 2) in vehicle length ranges $L$ as:

$$
\begin{gathered}
W_{F B} \in[1,1.5] L ; L_{F B} \in[2,2.5] L ; \quad F_{F B F} \in[1.5,2] L ; \\
\left(L_{F B F}-C G_{x}\right) \in[0.5,1] L ; \quad H_{F B} \in[0.3,0.5] L ;
\end{gathered}
$$

In the simulation, the fluid is air, under normal conditions. In Table 1, the set of simulation boundary conditions and values is presented. The mesh properties must satisfy certain flow constraints in order to model flow development correctly. Our simulation task began with setting up many mesh tests to determine the most convenient set of mesh parameters (Table 4). Tests were conducted to check for the convergence (value of residuals) of the method, as well as the convergence of drag and lift coefficients. Drag and lift coefficients behave in orthogonal way regarding the mesh setup. We can distinguish two types of mesh.x

Table 1. Simulation boundary conditions

\begin{tabular}{ll}
\hline Inlet & posed on face $x>0.15 \mathrm{~m} / \mathrm{s}$, turbulence intensity $5 \%$ \\
\hline Outlet & posed on face $x<0$ \\
\hline Vehicle & wall type - no-Slip \\
\hline Road & wall type - noShearStress, \\
& posed on floor/road faces $(z=0)$ \\
\hline Symmetry & posed on left, right and top face \\
\hline
\end{tabular}

If a mesh is good for drag, it is not entirely true that is also acceptable for lift (and vice-versa). There is always interplay in a mesh setup to achieve good convergence properties in both directions (drag and lift). Comparison of a few mesh types showed that the convergence of a CFX numerical method is highly sensitive to the boundary layer mesh (BLM) type. Therefore, mesh construction for flow phenomena strongly depends on the BLM setup. One of the pillars in the setup of boundary layer parameters is the dimensionless wall distance $Y^{+}$, which controls the boundary mesh quality. A detailed explanation of this phenomenon can be found in [12]. In order to obtain the best mesh type, two inflation models/algorithms for boundary layer mesh generation were analyzed: FLT and FAR.

a)

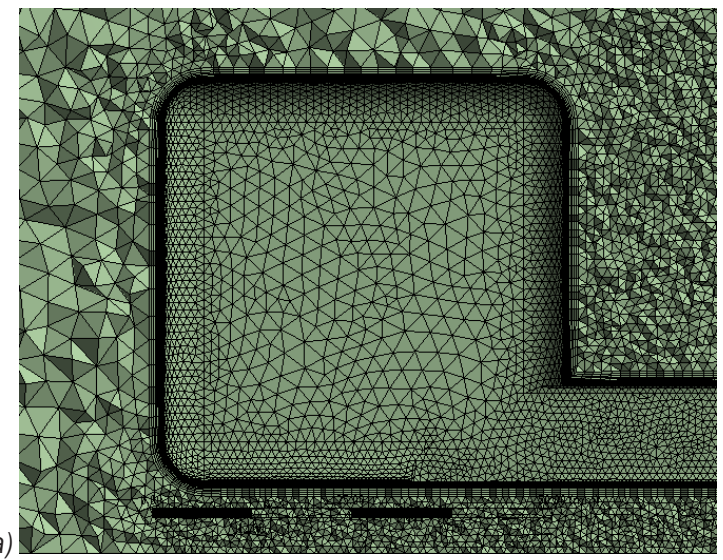

b)

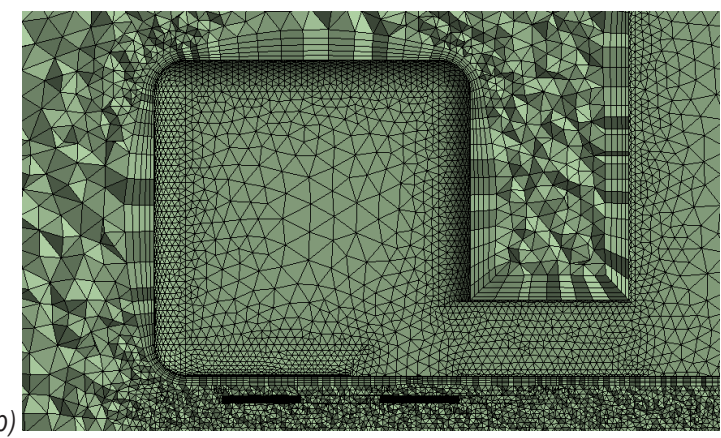

Fig. 4. Mesh configuration; a) FLTO3, and b) FARO2

In Table 3, we present data that were constant within all mesh tests. Those parameters were constant for all simulation experiments and for all different mesh types. To find a reasonable mesh capable of sustaining convergence properties for drag and lift coefficients, seven mesh parameters were chosen to be varied. Table 3 presents different parameters used to create relatively different meshes.

The difference between the two inflation models, FLT and FAR, is clearly explained graphically in Fig. 4. The FLT algorithm (Fig. 4a) expands the boundary layer mesh parallel to the vehicle geometry, and the mesh uniformity is clearly visible. In contrast, the FAR algorithm (Fig. 4b) creates a boundary layer mesh in which cell height is dependent on the size of the face cell element attached to the vehicle surface. 
The mesh is no longer uniform in the tangent direction of vehicle geometry.

a)
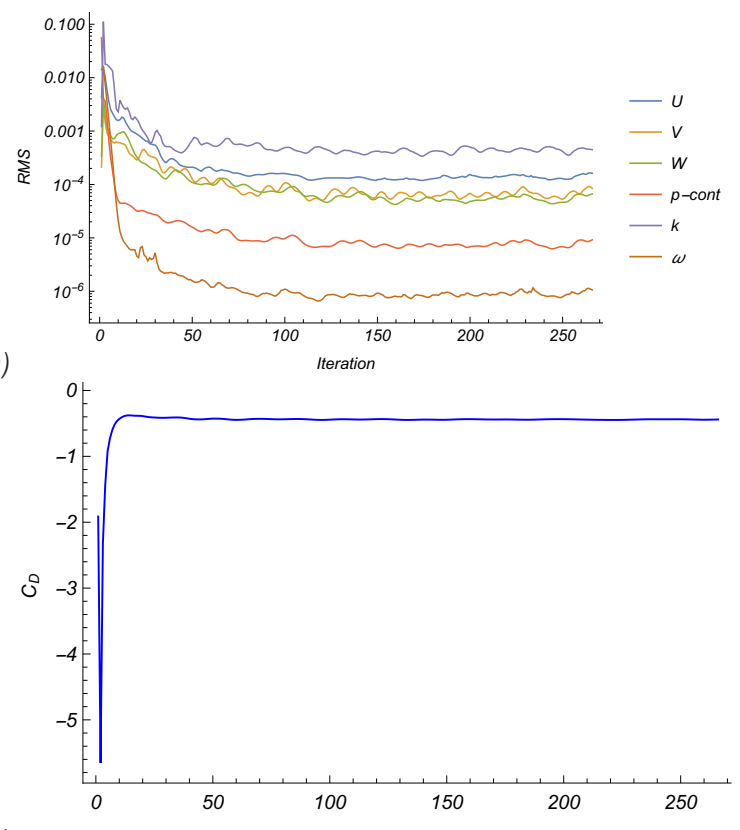

b)

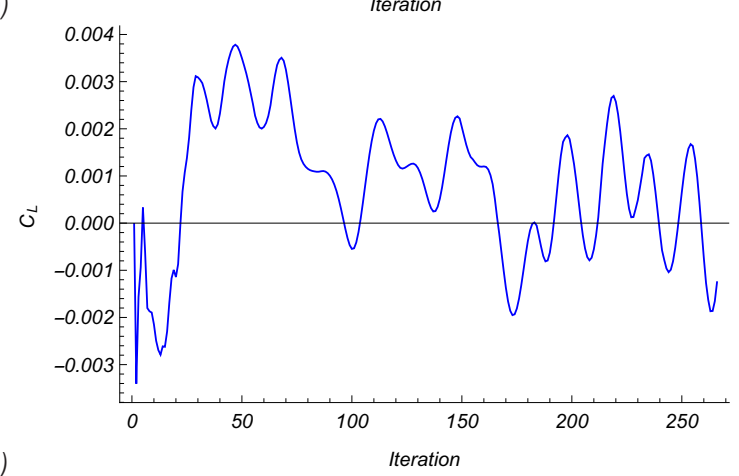

Fig. 5. Convergence results for mesh FLT-03;

a) residuals, b) drag coefficient and c) lift coefficient

In Table 3, the results obtained with the FAR algorithm show that its inflation is considerably better than that of the FLT with respect to the SST turbulent model. This was unexpected in the simulation tests. We taught that nice uniform BLM should provide stable and more accurate results. Simulation tests for all meshes with a yaw angle of $\psi=0^{\circ}$ were stopped for the condition controlling residuals $\left(\mathrm{RMS} \leq 10^{-5}\right)$ or number of maximal iterations $\left(\mathrm{N}_{\max } \leq 450\right)$. If a method did not converge (RMS condition) within $\mathrm{N}_{\max }$ iterations, it was considered not convergent. In Figs. 5 and 6, two different cases can be observed. Convergence results (RMS at Figs. 5a and 6a) show convergence of velocity components $(u, v, w)$, pressure $(p)$ and $k$-omega SST turbulence model variables $(k, \omega)$.

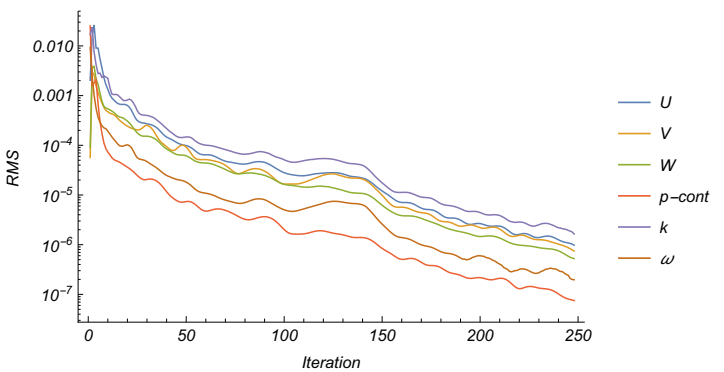

a)

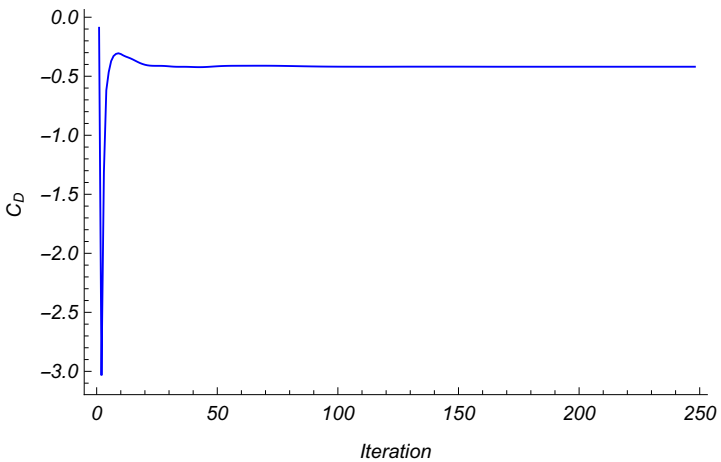

b)

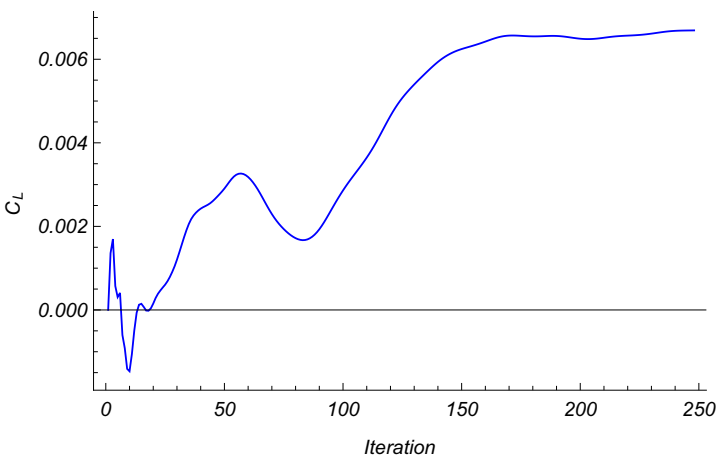

Fig. 6. Convergence results for mesh FAR-02; a) residuals, b) drag coefficient, c) lift coefficient

Convergence results (RMS) for the mesh type FLT-03 (Fig. 5a) clearly show that the method did not converge, and that the lift coefficient was highly unstable. The computed flow with an FLT mesh behaves in an unusual manner. In contrast, in Fig. 6, we show results for the mesh type FAR-02, with good method convergence and very good aerodynamic coefficient convergence. In Table 4 and Fig. 7, simulation results for drag and lift coefficients with respect to different mesh types are compared. They show clear evidence that the FAR mesh type has the desired characteristics. FAR mesh type results are spread in a narrow band, and show very small fluctuations with respect to different mesh parameters. The stability region of aerodynamic coefficients with respect to different mesh properties is, in the case of the FAR mesh, very confined. The stable properties of the FAR mesh type are even more evident, because the mesh parameters were varied over a wide interval. 
The cause of such differences in FLT and FAR mesh types probably originates in the underlying turbulent model. Turbulent models are very sensitive to the type of boundary mesh.

The SST model performs much better with a FAR mesh type, because of the nature of its near-wall fluid turbulent motion filtering [9] and [12]. An FLT mesh type shows a completely different picture. Variations in the mesh properties result in vast fluctuations in the aerodynamic coefficients. It can be concluded that the FAR mesh type must be used to simulate external aerodynamics for the SST model.

Comparing all results (Table 4 and Fig. 7) for different mesh tests, it can be observed that mesh FAR02 is a good candidate and has stable computational properties in simulations of the present external aerodynamic problem. The mesh is not too dense (i.e., it will not cause slow computations) and not too coarse (i.e., it will not produce the wrong coefficient results). Mesh FAR-02 was used in all computations in the analysis of aerodynamic coefficients. The aerodynamic coefficients are the non-dimensional representation of forces and moments of flow acting on a body (in our case, a vehicle).

Table 2. Constant mesh data

\begin{tabular}{lccc}
\hline Name & Sign & Unit & Value \\
\hline far velocity & $U_{\infty}$ & $\mathrm{m} / \mathrm{s}$ & 15 \\
\hline inlet turbulent intensity & & $\%$ & 5 \\
\hline boundary layer height & $H_{B L}$ & $\mathrm{~m}$ & $3 \cdot 10^{-3}$ \\
\hline
\end{tabular}

Table 3. Mesh parameters; length/height dimensions are in millimeters

\begin{tabular}{lccccccc}
\hline $\begin{array}{c}\text { mesh } \\
\text { type }\end{array}$ & domain & $\begin{array}{c}\text { fine } \\
\text { box }\end{array}$ & $H_{1}$ & $Y^{+}$ & $\begin{array}{c}\text { inflation } \\
\text { model }\end{array}$ & layers & GR \\
\hline FLT-01 & 20 & 8 & 0.6 & 50 & FLT & 5 & 1.2 \\
\hline FLT-02 & 20 & 6 & 0.1 & 10 & FLT & 10 & 1.2 \\
\hline FLT-03 & 20 & 4 & 0.01 & 1 & FLT & 20 & 1.2 \\
\hline FLT-04 & 20 & 4 & 0.001 & 0.085 & FLT & 35 & 1.2 \\
\hline FLT-05 & 20 & 3.5 & 0.0005 & 0.04 & FLT & 35 & 1.2 \\
\hline FAR-01 & 20 & 2 & - & - & FAR & 5 & 1.2 \\
\hline FAR-02 & 15 & 5 & - & - & FAR & 5 & 1.2 \\
\hline FAR-03 & 15 & 7 & - & - & FAR & 5 & 1.2 \\
\hline Domain & cell size in basic domain & & & \\
FineBox & cell size in FineBox subdomain \\
$H_{1}$ & height of first boundary layer cell (attached to the wall) \\
$Y^{+}$ & $\begin{array}{l}\text { calculated dimensionless wall distance with equations } \\
\text { described in [12] }\end{array}$ \\
Inflation \\
Layers \\
GR inflation models were used FLT and FAR \\
the number of layers in boundary layer mesh zone \\
the boundary layer mesh growth rate
\end{tabular}

Let us first define the aerodynamic force coefficient [6]:

$$
C_{F}=\frac{F}{\frac{1}{2} \rho A U^{2}},
$$

where $F$ is the force magnitude in a specific direction, $\rho$ is fluid density, $U$ is apparent wind velocity, and $A$ is the projected frontal vehicle area. When flow forces act on a vehicle, external moments also develop, due to the non-symmetry of vehicle geometry. As for the force aerodynamic coefficients, the aerodynamic coefficient for moment [6] can be defined, and is described by:

$$
C_{M}=\frac{M}{\frac{1}{2} \rho A h U^{2}},
$$

where $M$ is the magnitude of the moment with respect to a specific axis and $h$ is a length scale parameter. Different authors choose different length scale parameters $h$ (for example vehicle height, vehicle length, or height of the center of gravity (CG)). We fix $h$ (Fig.s 1 and 8 ) to be the height of the $\mathrm{CG}\left(H_{\mathrm{CG}}\right)$ in such a way that the pitch moment coefficient is 0 for yaw angle $\psi=0^{\circ}$.

In, Fig. 3 we show an illustration of the coordinate system orientation, vehicle rotation, and definition of force and moment coefficients in space. The coordinate system is always attached to the vehicle, and the apparent wind direction is described by yaw angle $\psi$. Simulation results were obtained from a fixed tunnel simulation experiment, with vehicle geometry being rotated around the $z$ axis passing through the CG. CFD simulation results of forces and moments were transformed from the tunnel coordinate system into the vehicle coordinate system (Fig. 3). The force aerodynamic coefficients are $C_{D}$ (drag $-x$ axis), $C_{S}$ (side $-y$ axis), and $C_{L}$ (lift $-z$ axis). The moment aerodynamic coefficients are $C_{R}$ (roll - over the $x$ axis), $C_{P}$ (pitch - over the $y$ axis), and $C_{Y}$ (yaw - over the $z$ axis). We must emphasize that the coefficients are not constant, and can change significantly depending on the apparent wind angle $\psi$. Data for the aerodynamic coefficients obtained from CFD calculation are discrete point set values. For the stability analysis, we must represent the aerodynamic coefficients in a functional form. How this can be achieved is explained in the next section.

Data fitting [14] is a science in its own right. In this section, a comparison of different fit models for the aerodynamic coefficients is presented. We introduce a new fit model to account for asymmetric 
cases. The asymmetry in the results, shown in Fig. 9, motivated us in the search for a periodic but asymmetric function that can accurately fit the obtained computational data. The asymmetry can be incorporated into a combination of linear and cyclic functions. We propose the following piece-wise fit function:

$$
g(x)=\left\{\begin{array}{l}
y_{L}+k_{L} x+A_{L} \sin \left(\omega_{L}+\delta_{L}\right) \\
y_{R}+k_{R} x+A_{R} \sin \left(\omega_{R}+\delta_{R}\right)
\end{array},\right.
$$

where index $L$ stands for $x \in[0, \pi / 2]$ and index $R$ stands for $x \in[\pi / 2, \pi]$. In Eq. (3), $x$ is the apparent wind angle $\psi$. Function $g(x)$ is continuous and must satisfy the continuity condition at point $x=\pi / 2$. Function $g(x)$ must have an equal left and right limit at the mentioned point:

$$
\lim _{x \uparrow \pi / 2} g(x)=\lim _{x \downarrow \pi / 2} g(x) .
$$

Eq. (3) can account for asymmetry with respect to the point $x=\pi / 2$. The coefficients can be expected to behave in a nonsymmetrical way due to the nonsymmetrical vehicle geometry. In most stability analysis cases, symmetrical relationships are used [4], [5] and [7], and we compare them with our simulation results. In order to complete the functional form of the aerodynamic coefficient, a CFD analysis must be performed for different apparent wind angles. In our case, this was accomplished for an angle interval from $0^{\circ}$ to $180^{\circ}$, with constant angle increments of $10^{\circ}$. Smaller angle increments will probably not improve the accuracy of the chosen function form (Eq. (3)).

Computations were done in parallel (16 cores) and were completed within $10 \mathrm{~d}$. For more complex vehicle geometries, computational time would probably multiple of 10 days. There is always interplay between computational time and computational accuracy. This dilemma was partially apparent in the mesh analysis section. Deeper mesh analysis for a variety of vehicle types is needed to be completely confident. In the calculation of aerodynamic coefficients (Eqs. (1) and (2)) we provide values for reference area $A$ (the projection of the frontal truck area onto the " $y z$ " plane), free stream velocity $U$, fluid density $\rho$, and moment arm characteristic length $h$. The data used in the present simulations are shown in Table 1. CFD simulation results are obtained for pressure (index $\mathrm{P}$ ) and viscous (index $\mathrm{V}$ ) forces and moments $\left(\mathbf{F}_{\mathrm{P}}, \mathbf{F}_{\mathrm{V}}, \mathbf{M}_{\mathrm{P}}, \mathbf{M}_{\mathrm{V}}\right)$ for different $\psi$. The present analysis does not focus on a separate analysis of pressure and viscous effects, but its magnitude and partial forces and moments must be summed:

$$
\begin{gathered}
\mathbf{F}=\mathbf{F}_{\mathrm{P}}+\mathbf{F}_{\mathrm{V}}=\left(F_{x}, F_{y}, F_{z}\right), \\
\mathbf{M}=\mathbf{M}_{\mathrm{P}}+\mathbf{M}_{\mathrm{V}}=\left(M_{x}, M_{y}, M_{z}\right) .
\end{gathered}
$$

Forces and moments are provided for all yaw angles. In Fig. 9, the results for aerodynamic coefficients are shown, and are compared for different fit models. Simulation data are compared with experimental data [8] and [15] obtained in a wind tunnel experiment. A comparison was performed for different inlet turbulent intensities and different measurements of the same object, as is investigated in the present study. The simulation data show very good agreement with the experimental data, except for pitch moment coefficient (CP). Experimental data from [8] and [15] show the same unusual CP behavior. The most probable cause of such unusual results lies in the sensitivity of the CP coefficient. Pitch moment can jump an order equal to its value for changes in the apparent window direction $\psi$, causing huge variations in CP. Given such fluctuations, it is almost impossible in a functional sense to correctly predict CP behavior. In Fig. 9, we show four fit functions: Fit-B1 are taken from [7], Fit-B2 are taken from [5], Fit-B3 are taken

Table 4. Mesh test simulation parameters

\begin{tabular}{rrrrrrrrr}
\hline $\mathrm{i}$ & mesh type & \# Cells & \# iter & Time/iter & CDrag & CLift & RMS & Converged \\
\hline 1 & FLT-01 & $4,674,278$ & 145 & $00: 00: 29$ & -0.426 & -0.0337 & $10-5$ & N0 \\
\hline 2 & FLT-02 & $6,308,164$ & 765 & $00: 00: 42$ & -0.430 & -0.0140 & $10-5$ & N0 \\
\hline 3 & FLT-03 & $11,120,526$ & 266 & $00: 01: 27$ & -0.440 & -0.00204 & $10-5$ & N0 \\
\hline 4 & FLT-04 & $11,898,277$ & 177 & $00: 01: 49$ & -0.464 & 0.0297 & $10-5$ & N0 \\
\hline 5 & FLT-05 & $14,724,983$ & 3126 & $00: 01: 04$ & -0.446 & 0.0249 & $10-5$ & N0 \\
\hline 6 & FAR-01 & $3,227,637$ & 204 & $00: 00: 20$ & -0.427 & -0.0227 & $10-5$ & YES \\
\hline 7 & FAR-02 & $8,621,470$ & 1546 & $00: 00: 51$ & -0.419 & -0.0214 & $10-5$ & YES \\
\hline 8 & FAR-03 & $16,081,773$ & 1386 & $00: 02: 09$ & -0.419 & -0.0218 & $10-5$ & YES \\
\hline 9 & FAR-01 & $3,227,637$ & 536 & $00: 00: 20$ & -0.427 & -0.0238 & $10-6$ & YES \\
\hline 10 & FAR-02 & $8,621,470$ & 2486 & $00: 00: 50$ & -0.419 & -0.0227 & $10-6$ & YES \\
\hline 11 & FAR-03 & $16,081,773$ & 261 & $00: 02: 12$ & -0.419 & -0.0237 & $10-6$ & YES \\
\hline
\end{tabular}



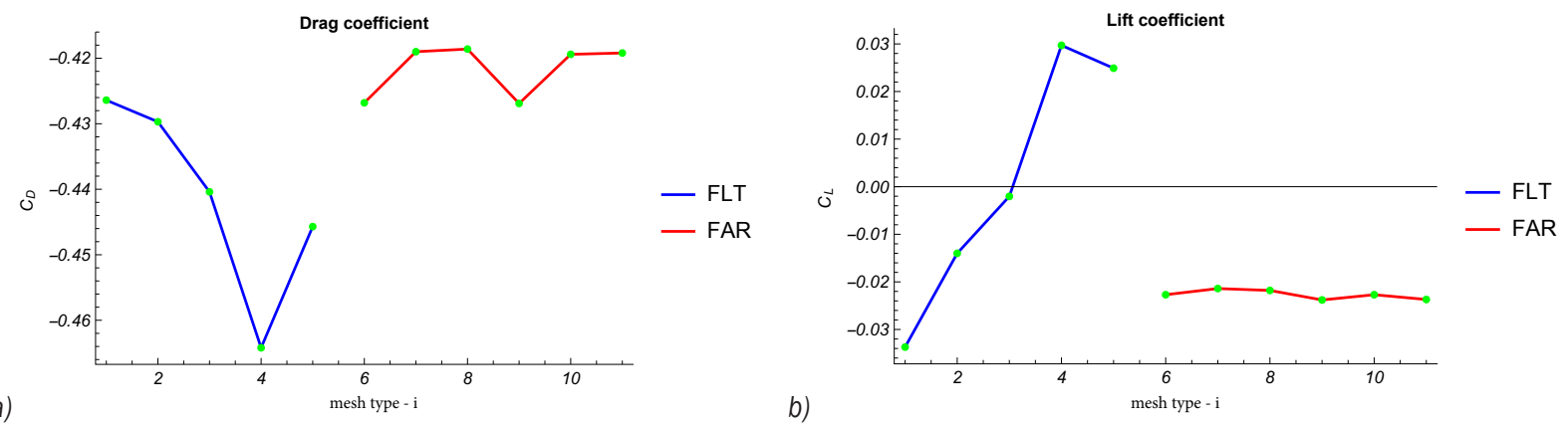

Fig. 7. Plot of a) drag and b) lift coefficients with respect to mesh type (Table 4)

from [4] and $g(x)$ is the function in Eq. (3). With $S_{1}$, we show points obtained from the CFD simulations. The CFD results $\left(S_{1}\right)$ fit almost perfectly and follow the same trends as experimental data, except for $C_{P}$.

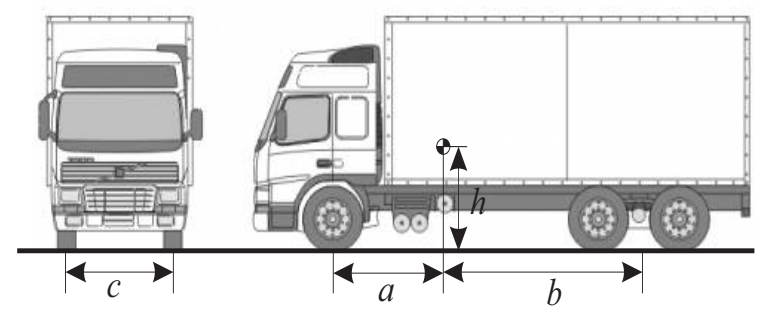

Fig. 8. Vehicle dimensions

As previously mentioned, the reason behind the large fluctuations and fit procedure for different fit functions produce evidently different behavior. In the present CFD analysis, simulations for angles greater than $90^{\circ}$ were performed, and fill the gap in the data set compared to the experimental data in [8] and [15]. The CFD results show significant data shifting and asymmetry with respect to $\psi$. Reference fit functions $F i t-B_{1}$, Fit- $B_{2}$, and Fit- $B_{3}$ show very good fitting properties, although they have fewer degrees of freedom compared to the proposed function described in Eq. (3). Relationships similar to Fit- $B_{1}$ are mostly needed in analytic studies of crosswind effects, because of their simple but expressive form. In the case of coefficient $C_{L}$, the second minima (near $100^{\circ}$ ) can be observed, and was not predicted in the CFD analysis. Instead, it emerged as a fit result. One would expect that the force coefficients must reassemble symmetry around $90^{\circ}$; in the case of $C_{L}$, this occurred only with fitting result. Such evidence is not standard, and must be verified with additional CFD studies. As for the $C_{P}$, the oscillations are smeared, and the resulting fit function behaves very theoretically.

However, $C_{L}$ and $C_{P}$ are always somewhat "problematic" coefficients. The fit results in Fig. 9 are very informative, and show good agreement with the CFD results, except in the case of these "problematic" coefficients. We can conclude that the sets of fit functions presented in Fit- $B_{1}$, Fit- $B_{2}$ and Fit- $B_{3}$ have significant informative value, remembering that these are defined with only one or two coefficients.

We have compared four different sets of fit functions. The set of fit functions in Eq. (3) accommodates the results in a promising way. However, to be confident of the chosen fit function, it is highly desirable to test its form on a set of different types of vehicles, and verify its structure. This is reserved for future research.

\section{AERODYNAMIC STABILITY ANALYSIS}

This section covers the aerodynamic stability analysis of the vehicle studied in Sect. 1. An essential element of this method is the relationship between wind speed, truck speed, and aerodynamic forces for a particular vehicle and site.

Table 5. Geometry and wind parameters

\begin{tabular}{lccc}
\hline Name & Symbol & Unit & Value \\
\hline air density & $\rho$ & $\mathrm{kg} / \mathrm{m}^{3}$ & 1.185 \\
\hline front area & $A$ & $\mathrm{~m}^{2}$ & 0.00405 \\
\hline velocity & $U$ & $\mathrm{~m} / \mathrm{s}$ & 15.0 \\
\hline moment arm & $h$ & $\mathrm{~m}$ & 0.068396 \\
\hline mass & $m$ & $\mathrm{~kg}$ & 10000 \\
\hline
\end{tabular}

Traditionally, these aerodynamic characteristics have been studied with scaled-model wind tunnel tests [16] and [17]. In contrast, this study uses the CFD method described in Sect. 1 for these purposes. Experiments are costly and the required special equipment is not widely available, which poses a serious problem for wind tunnel experiments. Recent and similar works [1] and [18] to [20] performed a similar analysis under different circumstances in a 
crosswind situation. In this study, we use the stability criteria relationship derived in [2] to analyze three crosswind accident types: rollover, side-slip or lateralslip, and rotation.

\subsection{Rollover}

The first indication of possible vehicle rollover occurs when a wheel loses contact with the road.

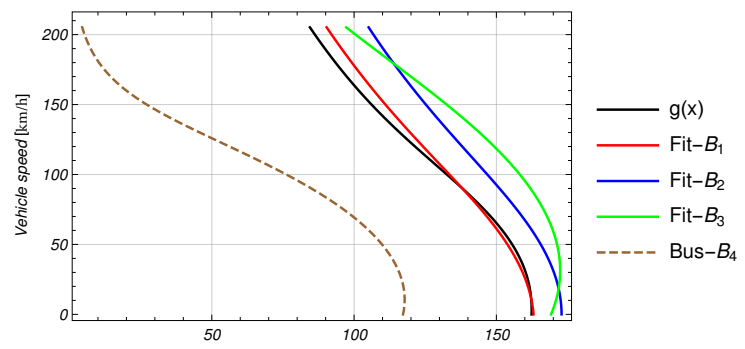

a)

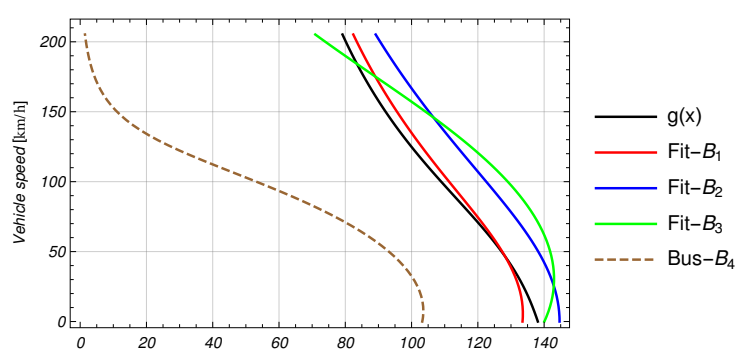

b)

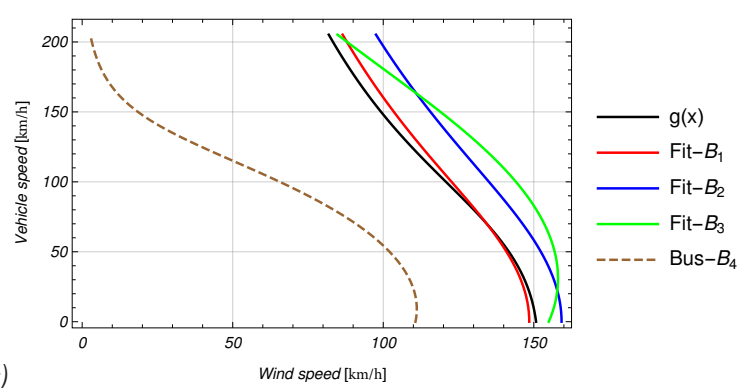

Fig. 10. Solution of a) $V_{\text {loose } 1}$, b) $V_{\text {loose2 }}$, and c) $V_{\text {roolover }}$

To determine which wheel will lose contact, we use Eqs. (4) and (5) (derived in [2]) to calculate the critical apparent wind speed magnitude for the front $\left(V_{\text {loose1 }}\right)$ and rear $\left(V_{\text {loose2 }}\right)$ wheels. If both wheels lose contact simultaneously, then we calculate critical velocity with Eq. (6). The rollover results are shown in Fig. 10. In addition to fit results, the comparison to the coefficients defined in [4] for the Leyland Atlantean bus are shown with the label Bus- $B_{4}$.

$$
V_{\text {loosel }}=\sqrt{\frac{2 m g}{\rho A} \frac{b c}{h(a+b)\left(C_{S}+C_{R}\right)+h c\left(C_{D}+C_{P}\right)+b c C_{L}}}
$$

$$
\begin{gathered}
V_{\text {loose } 2}=\sqrt{\frac{2 m g}{\rho A} \frac{a c}{h(a+b)\left(C_{S}+C_{R}\right)-h c\left(C_{D}+C_{P}\right)+a c C_{L}}}, \\
V_{\text {rollover }}=\sqrt{\frac{2 m g}{\rho A} \frac{c}{2 h\left(C_{S}+C_{R}\right)+c C_{L}}} .
\end{gathered}
$$

\subsection{Rotation}

The vehicle reaches a rotation condition if the side forces on one of its axles reach the friction limit. Eq. (7) $\left(V_{\text {slip } 1}\right)$, derived in [2], describes the magnitude of the critical apparent wind speed that will slip the front axle wheels. Eq. (8) $\left(V_{\text {slip2 }}\right)$, derived in [2], describes the magnitude of the critical apparent wind speed for which the back axle wheels lose contact. The critical condition rotation results are shown in Fig. 11.
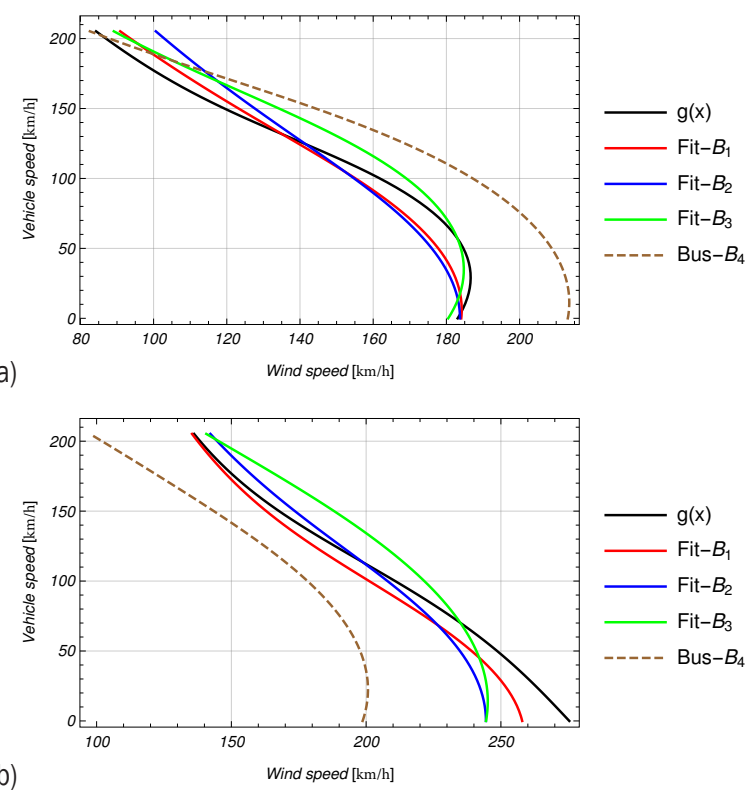

Fig. 11. Solution of a) $V_{\text {slip } 1}$ and b) $V_{\text {slip2 }}$

$$
\begin{aligned}
& V_{\text {sipp }}=\sqrt{\frac{2 m g}{\rho A} \frac{\mu_{1} b}{b C_{S}+h\left[C_{Y}-q^{\prime}\left(C_{S}+C_{R}\right)\right]+\mu_{1}\left[b C_{L}+h\left(C_{D}+C_{P}\right)\right]}},(7) \\
& V_{\text {slipp }}=\sqrt{\frac{2 m g}{\rho A} \frac{\mu_{2} a}{a C_{S}-h\left[C_{Y}-q^{\prime}\left(C_{S}+C_{R}\right)\right]+\mu_{2}\left[a C_{L}-h\left(C_{D}+C_{P}\right)\right]}} .(8)
\end{aligned}
$$

\subsection{Side Slip or Lateral Slip}

The side-slip or lateral-slip criterion is reached when all vehicle wheels simultaneously reach the friction limit from sideway force. Eq. (9), derived in [2], describes the relationship for the critical apparent 
wind speed for side-slip and. The results of critical conditions can be seen in Fig. 12.

$V_{\text {sidestip }}=\sqrt{\frac{2 m g}{\rho A} \frac{a \mu_{2}+b \mu_{1}}{(a+b) C_{S}+\left(a \mu_{2}+b \mu_{1}\right) C_{L}+\left(\mu_{1}-\mu_{2}\right) h\left(C_{D}+C_{P}\right)}}$.

For all three mentioned cases, the relationship between vehicle and wind speed is implicit, and requires a numerical solution. The most severe and fatal situations occur for cases where the apparent wind blows perpendicularly to the vehicle.

\section{CONCLUSIONS}

In this study, a CFD analysis of vehicle external aerodynamics is compared with experimental data for a crosswind situation. The primary goal of the present numerical analysis is the analysis of vehicle stability under crosswind influence. A traditional analysis of vehicle stability under wind influence using a multibody approach requires only aerodynamic coefficients to set up the external forces problem. The analysis of crosswind conditions under which a vehicle will roll over, side-slip, or rotate is highly sensitive to wind direction (external force direction). To derive accurate conditions, we must provide relationships for aerodynamic coefficients that are dependent on the direction of external force. Such information is almost never available to researchers, and this was the primary goal of the present study.

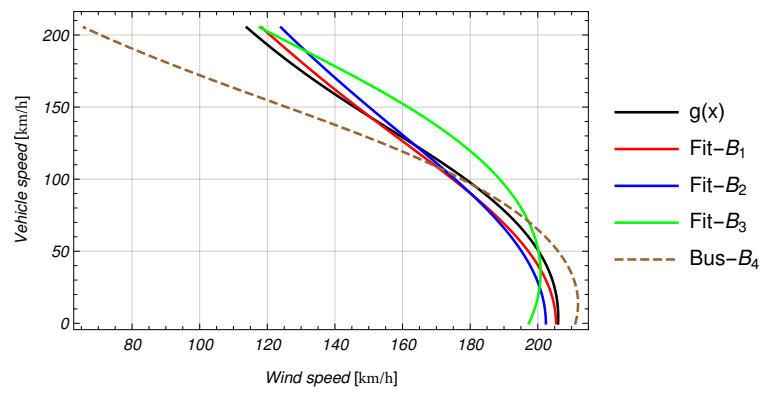

Fig. 12. Solution of $V_{\text {side-slip }}$

We describe a complete framework for the calculation of aerodynamic coefficients under high crosswinds. First, the mesh type is analyzed. Later, a CFD analysis is described, in order to provide accurate results. Finally, simulation results are compared to experimental data [8] and [15]. The results show good agreement between the experiments and simulations, except for critical cases in which the experimental data have no rational explanation. One important question is: which function should we use to accurately fit the simulation data? The simulation showed that there are significant discrepancies from symmetric geometrical situation. We proposed a piece-wise function $g(x)$ Eq. (3) to account for data shifting and asymmetry. All simulation data were fit to $g(x)$, and the results are shown in Fig. 9. The results match the simulation data well. In particular, they reveal some hidden behavior with respect to lift and pitch coefficients. Reference fit functions $F i t-B_{1}$, Fit- $B_{2}$, and $F i t-B_{3}$ show very good agreement, although they incorporate significantly fewer degrees of freedom. In the application, the results obtained with fit models were used to estimate critical aerodynamic stability parameters. Rollover, rotation, and side-slip results were obtained. As expected, the results show some differences depending on the fit model. The most important data are the lower bound predictions for critical speeds. A general observation is that there is a lower bound path defined within the family of fit models. This marginal path can be taken as the worst-case scenario for critical windvehicle speeds, and can be used in the analysis of wind safety regulations.

\section{REFERENCES}

[1] Choi, H., Lee, J., Park, H. (2014). Aerodynamics of Heavy Vehicles. Annual Review of Fluid Mechanics, vol.46, n. 1, p. 441-468, D0I:10.1146/annurev-fluid-011212-140616.

[2] Batista, M., Perkovič, M. (2014). A simple static analysis of moving road vehicle under crosswind. Journal of Wind Engineering and Industrial Aerodynamics, vol. 128, p. 105113, D0I:10.1016/j.jweia.2014.02.009.

[3] Hucho, W.H. (2013). Aerodynamics of road vehicles: from fluid mechanics to vehicle engineering. Elsevier.

[4] Baker, C. (1986). A simplified analysis of various types of wind-induced road vehicle accidents. Journal of Wind Engineering and Industrial Aerodynamics, vol. 22, n. 1, p. 6985, D0I:10.1016/0167-6105(86)90012-7.

[5] Baker, C. (1988). High sided articulated road vehicles in strong cross winds. Journal of Wind Engineering and Industrial Aerodynamics, vol. 31, n. 1, p. 67-85, D0l:10.1016/01676105(88)90188-2.

[6] Baker, C. (1991). Ground vehicles in high cross winds part l: steady aerodynamic forces. Journal of Fluids and Structures, vol. 5, n.1, p. 69-90, D0l:10.1016/0889-9746(91)80012-3.

[7] Snæbjörnsson, J.T., C. Baker, Sigbjörnsson, R. (2007). Probabilistic assessment of road vehicle safety in windy environments. Journal of Wind Engineering and Industrial Aerodynamics, vol. 95, no. 5, p. 1445-1462, D0l:10.1016/j. jweia.2007.02.020.

[8] Coleman, S., Baker C. (1994). An experimental study of the aerodynamic behaviour of high sided lorries in cross winds. Journal of Wind Engineering and Industrial Aerodynamics, vol. 53, no. 3, p. 401-429, Dol:10.1016/0167-6105(94)90093-0.

[9] Menter, F.R. (2009). Review of the shear-stress transport turbulence model experience from an industrial perspective. 
International Journal of Computational Fluid Dynamics, vol. 23, n. 4, p. 305-316, Dol:10.1080/10618560902773387.

[10] Ferziger, J.H., Perić, M. (2012). Computational Methods for Fluid Dynamics. Springer Science \& Business Media.

[11] Patankar, S. (1980). Numerical Heat Transfer and Fluid Flow. CRC press.

[12] Pope, S.B. (2001). Turbulent Flows. IOP Publishing, D0I:10.1088/0957-0233/12/11/705.

[13] Schlichting, H., Gersten, K. (2003). Boundary-Layer Theory. Springer Science \& Business Media.

[14] Quarteroni, A., Sacco, R., Saleri, F. (2010). Numerical Mathematics. Springer Science \& Business Media.

[15] Baker, C.J. (1987). Measures to Control Vehicle Movement at Exposed Sites during Windy Periods. Journal of Wind Engineering and Industrial Aerodynamics, vol. 25, n. 2, p. 151-161, D0I:10.1016/0167-6105(87)90013-4.

[16] Cheli, F., Corradi, R., Sabbioni, E., Tomasini, G., (2011). Wind tunnel tests on heavy road vehicles: Cross wind induced loads-Part 1. Journal of Wind Engineering and
Industrial Aerodynamics, vol. 99, n. 10, p. 1000-1010, DOI:10.1016/0167-6105(87)90013-4.

[17] Cheli, F., Corradi, R., Sabbioni, E., Tomasini, G., (2011). Wind tunnel tests on heavy road vehicles: Cross wind induced loads-Part 2. Journal of Wind Engineering and Industrial Aerodynamics, vol. 99, n. 10, p. 1011-1024, D0l:10.1016/j. jweia.2011.07.007.

[18] Bettle, J., Holloway, A.G.L., Venart, J.E.S. (2003). A computational study of the aerodynamic forces acting on a tractor-trailer vehicle on a bridge in cross-wind. Journal of Wind Engineering and Industrial Aerodynamics, vol. 91, n. 5, p. 573-592, DOI:10.1016/S0167-6105(02)00461-0.

[19] Cai, C.S., Hu, J., Chen, S., Han, Y., Zhang, W. and Kong, X. (2015). A coupled wind-vehicle-bridge system and its applications: a review. Wind and Structures, vol. 20, n. 2, p. 117-142, Dol:10.12989/was.2015.20.2.117.

[20] Iljaž, J., Škerget, L., Štrakl, M., Marn, J. (2016). Optimization of SAE Formula Rear Wing. Strojniški Vestnik-Journal of Mechanical Engineering, vol. 62. n. 5, p. 263-272, DOI:10.5545/sv-jme.2016.3240. 